\title{
Variable Focal Length Relative Orientation Calibration Method Research Based on p-h Algorithm
}

\author{
Yang Bin, Wang Yongjiao and Zhao Junmin \\ Institute of Computer Science and Engineering, Henan University of Urban \\ Construction, Pingdingshan 467036 \\ yb@hncj.edu.cn,wangyongjiao@126.com,zjm@hncj.edu.cn
}

\begin{abstract}
Aiming at the defects of traditional variable focal length relative orientation algorithm, such as strong dependence on initial value and poor convergence, the paper proposes a variable focal length relative orientation method based on $p$-h algorithm. In order to solve the defects caused by rotation matrix formed by trigonometric function, this method uses unit quaternion instead of trigonometric function to describe rotation matrix, solving algorithm parameters iteratively according to the least square principle. New method is optimized by $p$-h algorithm, and it established the corresponding solution model. The experimental results showed that the improved algorithm has the following characteristics: initial value weak dependence, fast convergence, adaptability for large angle, which solved multivaluedness and singularity problems.
\end{abstract}

Keywords: unit quaternion; $p-h$ algorithm; relative orientation; rotation matrix; variable focal length

\section{Introduction}

With the continuous development of photogrammetry and digital camera technology, the three-dimensional reconstruction technology of images has attracted more and more attention from domestic and foreign specialists. Three steps including extracting and matching feature points of images, camera calibration and building three-dimensional model are significant for three-dimensional reconstruction technology, among which digital camera calibration is one of the key technologies. In the process of image measurement and application of machine vision, the geometric model of camera imaging must be built in order to ascertain the mutual relation between the three-dimensional geometric position of a certain point on the subject and the position it locates on the image. The process of solving model parameters is called camera calibration [1].

For the past years, many camera calibration methods sprang up. In a broad sense, these methods can be classified into two types. The first type needs accurate three-dimensional fixed points. By making use of the three-dimensional fixed points, the equation set is established to acquire orientation elements of the camera. The advantage of the first type is all the internal and external parameters of the camera can be solved at the same time. The shortcoming is more accurate three-dimensional fixed points are needed and multivaluedness will be obtained in the resolving result. The RAC (Radial alignment constraint) camera calibration method [2] that Tsai proposed belongs to this type. Another type of methods is independent from three-dimensional fixed points. Parameters of the camera can be solved in accordance with the relation between images and the constraints of camera motion. Two images are needed in this type of methods and matching image pairs are available by matching feature points; then, parameters can be solved according to the relation between image pairs. The plane calibration algorithm [3] proposed by Zhang zhengyou is one of this type. The second type of methods also includes methods of fixed focus relative orientation and variable focus relative orientation. The fixed focus 
relative orientation method can only deal with images with the same focus, while variable focus relative orientation method has no such limitation. Both these two algorithms have strong dependence on the initial value. Before the algorithm is carried out, the unknown parameters should be evaluated and initiated first. Only when the evaluation value of the initiated parameters is close to the real experiment value, accurate calibration result can be worked out. If there is great difference between the evaluation value of the initiated parameters and the actual calibration value, especially when the rotation angle is overestimated, accurate result is impossible to be worked out even being tried several times. Therefore, this method needs further improvement.

Aiming at the defects of traditional relative orientation and variable focal length relative orientation algorithms, the paper proposes a variable focal length relative orientation method based on $\mathrm{p}$-h algorithm. In this algorithm, unit quaternion is used to form rotation matrix, and focal length is put into the process of relative orientation to obtain the solution. The application of $\mathrm{p}$-h algorithm can well deal with problems like parity, multivaluedness, singularity, strong dependence on initial value and misconvergence of wide angle, which are caused by rotation matrix of trigonometric function.

\section{Traditional Relative Orientation Algorithm}

\subsection{Concept and Calibration Parameter}

Relative orientation is actually a process of continuously reconstituting photographic rays of the left and right image and making the corresponding image rays of two images cross so as to obtain a spatial model proportional to the object model as well as solving relative orientation parameters to satisfy the above conditions [4]. Frequently used relative orientation includes relative orientation of separate image and relative orientation of continuous image. Relative orientation algorithm of separate image is subject to the photographic base, that is to say, it takes the photographic base level as the axle of the auxiliary space coordinate system and realizes relative orientation through angular motion of two images. The principle of relative orientation of continuous image is that it takes the left image as the benchmark and realizes relative orientation of image pairs through rectilinear motion and angular motion of the right image [5]. In this paper, relative orientation of continuous image pairs is adopted to improve variable focal length relative orientation algorithm.

The main work of digital photogrammetry is to calibrate the parameters of separate camera and camera pairs, and to build the imaging model with parameters of the actual calibration result. The objects of calibration include intrinsic parameters and extrinsic parameters [1]. There are three main extrinsic parameters, namely, the relative locations of principal image points (the perpendicular foot of principal optic axis on image level), and the vertical interval between the lens center and the image level. The intrinsic parameters, first calibrated in laboratory, reflect the inherent attribute of the camera and have nothing to do with photographic scene. The extrinsic parameters describe the spatial position relation between images during the imaging procedure. There are six extrinsic parameters, i.e., three line elements to describe the locations of lens center in geodetic coordinate system, and three angle elements to denote three separate parameters of rotation matrix. The extrinsic parameters are dependent on photographic scene, thus they need to be re-calibrated when the location or angle of the camera change during the shooting procedure [6].

\subsection{Calibration of Fixed and Variable Focal Length Algorithm}

Traditional relative orientation algorithms includes fixed focal length relative orientation and variable focal length relative orientation [7-8]. As for fixed focal length 
relative orientation calibration algorithm, there are five unknown parameters to be solved, namely $\varphi_{1,} \kappa_{1}, \varphi_{2}, \omega_{2}, \kappa_{2}$ or $B_{\gamma}, B_{2}, \varphi_{2}, \omega_{2,} \kappa_{2}$. This algorithm requires that the offset of the principal image points $x_{0}, y_{0}$ is small and ignorable, and the focal lengths of two cameras are equal, $f=f_{1}=f_{2}$. The variable focal length relative orientation calibration algorithm can deal with two pictures with different focal lengths, therefore there are six unknown parameters to be solved in this method, namely $\varphi, \omega, \kappa, \mu, \nu, f$ or $B_{y}, B_{z}, \varphi_{2}, \omega_{2}, \kappa_{2}, f$.

The calculation processes of these two algorithms are similar. In the first place, convert non-linear coplanarity equation (1) into a linear one by using of Taylor formula to get equation (3).

In the equation (1),

$$
\begin{aligned}
F & =\left|\begin{array}{lll}
B_{X} & B_{Y} & B_{Z} \\
X_{1} & Y_{1} & Z_{1} \\
X_{2} & Y_{2} & Z_{2}
\end{array}\right|=0 \\
& {\left[\begin{array}{l}
X \\
Y
\end{array}\right]=R\left[\begin{array}{l}
x
\end{array}\right] }
\end{aligned}
$$

$$
F=F_{0}+\frac{\partial F}{\partial \varphi} d \varphi+\frac{\partial F}{\partial \omega} d \omega+\frac{\partial F}{\partial \kappa} d \kappa+\frac{\partial F}{\partial B y} d B y+\frac{\partial F}{\partial B z} d B z+\frac{\partial F}{\partial f} d f=0
$$

Then, deduce equation (3) to get the resolving equation (4) of relative orientation algorithm.

$$
q=-\frac{X_{2} Y_{2}}{Z_{2}} N_{2} d \varphi-\left(Z_{2}+\frac{Y_{2}^{2}}{Z_{2}}\right) N_{2} d \omega+X_{2} N_{2} d \kappa+B_{X} d \mu-\frac{Y_{2}}{Z_{2}} B_{X} d v
$$

In the last step, solve these parameters by using iteration method in accordance with the principle of least square method to obtain the unknown parameters to be calibrated.

\section{Introduction to P-H Algorithm}

American scholar Pope proposed the idea of using quaternion tectonic rotation moment in photogrammetry analysis from the aspect of four-dimensional algebra [9]. On the basis of the above method, German scholar Hinsken deduced a set of algorithm, simply known as $\mathrm{p}$-h algorithm [10], which brought quaternion to extensive application in practical photogrammetry. P-H algorithm can effectively resolve the defects caused by rotation matrix based on trigonometric function.

The derivation process is as follows.

Four agebra parameters of unit quaternion satisfy equation (5):

$$
a^{2}+b^{2}+c^{2}+d^{2}=1
$$

Rotation matrix [11] formed by unit quaternion is as (6).

$$
R=\left[\begin{array}{ccc}
d^{2}+a^{2}-b^{2}-c^{2} & 2(a b+c d) & 2(a c-b d) \\
2(a b-c d) & d^{2}-a^{2}+b^{2}-c^{2} & 2(b c+a d) \\
2(a c+d b) & 2(b c-a d) & d^{2}-a^{2}-b^{2}+c^{2}
\end{array}\right]
$$

Through rotation matrix based on quaternion, it is easy to solve the three corresponding rotation angles formed by using trigonometric function. The relation is shown as formula (7).

$$
\begin{aligned}
& \varphi=\arctan \left(-R_{13} / R_{33}\right) \\
& \omega=\arcsin \left(-R_{23}\right) \\
& \kappa=\arctan \left(R_{21} / R_{22}\right)
\end{aligned}
$$


During conducting derivative optimization through $\mathrm{p}-\mathrm{h}$ algorithm, the directly estimated parameters are $W^{T}=\left(w_{1}, w_{2}, w_{3}\right)$ not $a, b, c, d$ in indirect compensating computation. The function relationship between them is as follows.

$$
\begin{gathered}
S_{W}=\left(\begin{array}{ccc}
0 & W_{3} & -W_{2} \\
-W_{3} & 0 & W_{1} \\
W_{2} & -W_{1} & 0
\end{array}\right) \\
d \boldsymbol{R}=S_{W} \boldsymbol{R}
\end{gathered}
$$

Perform the differential calculation of $\mathrm{R}$ and derive formula (10) by integrating formula (8) with formula (9).

$$
S_{W}=\frac{\partial R}{\partial d} R^{T} \Delta d+\frac{\partial R}{\partial a} R^{T} \Delta a+\frac{\partial R}{\partial b} R^{T} \Delta b+\frac{\partial R}{\partial c} R^{T} \Delta c
$$

The correction value can be obtained from formula (5).

$$
\Delta d: \quad \Delta d=-(a \Delta \mathrm{a}+b \Delta b+c \Delta c) / d
$$

The relation between the value of $\left[w_{1}, w_{2}, w_{3}\right]^{T}$ and the correction value $[\Delta a, \Delta b, \Delta c]^{T}$ is expressed in formula (12) derived from formula (10) and (11).

$$
\begin{aligned}
& \left(\begin{array}{l}
w_{1} \\
w_{2} \\
w_{3}
\end{array}\right)=\frac{2}{d}\left(\begin{array}{lll}
d^{2}+a^{2} & a b+d c & a c-d b \\
a b-d c & d^{2}+b^{2} & b c+d a \\
a c+d b & b c-d a & d^{2}+c^{2}
\end{array}\right)\left(\begin{array}{l}
\Delta a \\
\Delta b \\
\Delta c
\end{array}\right) \\
& \text { Here we set: } C=\frac{2}{d}\left(\begin{array}{lll}
d^{2}+a^{2} & a b+d c & a c-d b \\
a b-d c & d^{2}+b^{2} & b c+d a \\
a c+d b & b c-d a & d^{2}+c^{2}
\end{array}\right)
\end{aligned}
$$

Then we get the only inverse matrix of $\mathrm{C}$,

$$
C^{-1}=\frac{1}{2}\left(\begin{array}{ccc}
d & -c & b \\
c & d & -a \\
-b & a & d
\end{array}\right)
$$

It can be tested that $C C^{-1}=I, C^{-1} C=I$. For this reason, whatever values that the four parameters take, $C^{-1}$ always exists. That is to say, matrix $C$ is a nonsingular matrix, thus p$\mathrm{h}$ algorithm of rotation matrix formed by unit quaternion can avoid the problems of multivaluedness or singularity to achieve fast convergence.

\section{Calibration of Variable Focal Length Based on P-H Algorithm}

Defects exist in both traditional fixed focal length calibration algorithm and variable focal length calibration algorithm. In this paper, we propose a variable focal length calibration method based on $\mathrm{p}-\mathrm{h}$ algorithm. Variable focal length calibration algorithm is optimized by $\mathrm{p}-\mathrm{h}$ algorithm, and contributes to resolving the defects of traditional algorithms and enhancing the adaptation and stability as well.

\subsection{Parameters to be Calibrated}

When the focal lengths of the cameras are fixed and the focal lengths of two images are equal, then focal length $f$ can be calibrated as an intrinsic parameter. Therefore, there are five parameters to be calibrated in fixed focal length relative orientation algorithm. If the zoom camera is used, two pictures taken before and after zoom not always have the same intrinsic parameters. In addition to that, the focal length ratio of two images is hard to get. 
Under this situation; focal length ${ }^{f}$ should be added to the calculation formula as an unknown parameter. Therefore, there are six parameters to be calibrated in variable focal length relative orientation calibration algorithm. Two methods to deal with this situation, one is to set the assumed focal length $f_{1}$ as an unknown parameter; another one is to suppose $f_{2}=k^{*} f_{1}$ and set $k$ as the unknown parameter. The first method is adopted in this paper, thus the parameters to be calibrated are ${ }^{\varphi, \omega, \kappa, B_{y}, B_{z}, f_{2}}$.

\subsection{The Calculating Process}

Expand formula (1) through the multivariate function Taylor formula and obtain the linear function.

$$
\begin{aligned}
& d F=\left(Z_{1} X_{2}-X_{1} Z_{2}\right) d B_{y}+\left(X_{1} Y_{2}-X_{2} Y_{1}\right) d B_{z}+ \\
& \frac{\partial F}{\partial X_{2}} d X_{2}+\frac{\partial F}{\partial Y_{2}} d Y_{2}+\frac{\partial F}{\partial Z_{2}} d Z_{2}+\frac{\partial F}{\partial f} d f
\end{aligned}
$$

In the formula (14), $\quad \frac{\partial F}{\partial f}=\left|\begin{array}{ccc}B_{X} & B_{Y} & B_{Z} \\ 0 & 0 & -1 \\ X_{2} & Y_{2} & Z_{2}\end{array}\right|-\left|\begin{array}{ccc}B_{X} & B_{Y} & B_{Z} \\ X_{1} & Y_{1} & Z_{1} \\ -a_{1} & -a_{2} & -a_{3}\end{array}\right|$

In the calculating process, the rotation matrix formed by unit quaternion participates in operation. And in the solving procedure, three separate parameters take the place of parameters $a, b, c, d$, while the derivation process is optimized by $\mathrm{p}$-h algorithm.

The characters of orthogonal matrix $R R^{T}=E$ and $d R R^{T}+R d R^{T}=0$ prove that $d R R^{T}$ is an antisymmetric matrix, which can be expressed by matrix ${ }^{W_{\varepsilon}}$ formed by ${ }^{\varepsilon}=\left[\varepsilon_{1}, \varepsilon_{2}, \varepsilon_{3}\right]^{T}$, i.e. , $d R=W_{\varepsilon} R$. The matrix $W_{\varepsilon}$ is expressed as follows.

$$
W_{\varepsilon}=\left[\begin{array}{ccc}
0 & \varepsilon_{3} & -\varepsilon_{2} \\
-\varepsilon_{3} & 0 & \varepsilon_{1} \\
\varepsilon_{2} & -\varepsilon_{1} & 0
\end{array}\right]
$$

Formula (16) can be deduced, $\left[\begin{array}{l}d X_{2} \\ d Y_{2} \\ d Z_{2}\end{array}\right]=d R\left[\begin{array}{c}x_{2} \\ y_{2} \\ -f\end{array}\right]=W_{\varepsilon} R\left[\begin{array}{c}x_{2} \\ y_{2} \\ -f\end{array}\right]=W_{\varepsilon} S$

In the formula (16), $\quad \mathrm{S}=\left[\mathrm{X}_{2}, \mathrm{Y}_{2}, \mathrm{Z}_{2}\right]^{T}$

According to the extrinsic vector product theorem, $W_{\varepsilon} S=-W_{s} \varepsilon$

And in formula (17), $W_{s}=\left[\begin{array}{ccc}0 & Z_{2} & -Y_{2} \\ -Z_{2} & 0 & X_{2} \\ Y_{2} & -X_{2} & 0\end{array}\right]$

Substitute formula (17) and (18) into formula (16), and we get formula (19)

$$
\left[\begin{array}{l}
d X_{2} \\
d Y_{2} \\
d Z_{2}
\end{array}\right]=\left[\begin{array}{l}
Y_{2} w_{3}-Z_{2} w_{2} \\
Z_{2} w_{1}-X_{2} w_{3} \\
X_{2} w_{2}-Y_{2} w_{1}
\end{array}\right]
$$

Here, $w_{1}, w_{2}, w_{3}$ can directly participate in indirect adjustment calculation, and parameters $a, b, c, d$ in quaternion can be solved by using formula (12) to obtain the modified value and the iterated rotation matrix.

Combine formula (16) and (17) to get formula (20),

$$
\begin{aligned}
& V_{Q}=A_{1} Q_{p} B_{y}+A_{2} Q_{p} B_{z}+L d f+ \\
& B_{1} Q_{p} W_{1}+B_{2} Q_{p} W_{2}+B_{3} Q_{p} W_{3}-Q_{0}
\end{aligned}
$$




$$
\begin{aligned}
A_{1} & =X_{2} Z_{1}-Z_{2} X_{1}, A_{2}=Y_{2} X_{1}-X_{2} Y_{1} \\
L & =\left(\begin{array}{ccc}
B_{X} & B_{Y} & B_{Z} \\
0 & 0 & -1 \\
X_{2} & Y_{2} & Z_{2}
\end{array}|-| \begin{array}{ccc}
B_{X} & B_{Y} & B_{Z} \\
X_{1} & Y_{1} & Z_{1} \\
-c_{1} & -c_{2} & -c_{3}
\end{array}\right) /\left(X_{1} \cdot Z_{2}-X_{2} \cdot Z_{1}\right) \\
B_{1} & =Z_{2}\left(B_{z} X_{1}-Z_{1} B_{x}\right)+Y_{2}\left(B_{y} X_{1}-Y_{1} B_{x}\right) \\
\text { In formula (20), } B_{2} & =Z_{2}\left(B_{z} Y_{1}-B_{y} Z_{1}\right)+X_{2}\left(Y_{1} B_{x}-B_{y} X_{1}\right) \\
B_{3} & =Y_{2}\left(B_{y} Z_{1}-B_{z} Y_{1}\right)+X 2\left(Z_{1} B_{x}-B_{z} X_{1}\right) \\
Q_{p} & =\frac{-N_{2}}{\left(B_{x} Z_{1}-B_{z} X_{1}\right)}, \quad Q_{0}=N_{1} Y_{1}-N_{2} Y_{2}-B_{y} \\
N_{1} & =\frac{B_{x} Z_{2}-B_{z} X_{2}}{X_{1} Z_{2}-X_{2} Z_{1}}, \quad N_{2}=\frac{B_{x} Z_{1}-B_{z} X_{1}}{X_{1} Z_{2}-X_{2} Z_{1}}
\end{aligned}
$$

$\mathrm{N} 1$ and $\mathrm{N} 2$ are the projection coefficients of image points in auxiliary space coordinate system of two images. $X_{1}, Y_{1}, Z_{1}, X_{2}, Y_{2}, Z_{2}$ are the coordinates of a certain pixel point in auxiliary space coordinate system, and ${ }^{B_{x}, B_{y}, B_{z}}$ are the base line components.

Since there are six parameters to be calibrated, iteration solving can be conducted in accordance with the indirect adjustment principle of least square method when there are seven fixed points. Suppose $n$ pairs of image pairs are observed, and then $n$ error equations can be listed, the matrix form is as follows.

$$
\begin{gathered}
V=A X-U, \quad P=I \\
\text { And: } A=\left[A_{1} Q_{p}, A_{2} Q_{p}, L, B_{1} Q_{p}, B_{2} Q_{p}, B_{3} Q_{p}\right]^{T} \\
X=\left[B_{y}, B_{z}, f, \varepsilon_{1}, \varepsilon_{2}, \varepsilon_{3}\right]^{T}, U=Q_{0}
\end{gathered}
$$

The normal equation is $A^{T} P A X=A^{T} P U$

The solution of normal equation is $X=\left(A^{T} P A\right)^{-1} A^{T} P U$

The solution of the unknown parameters with relative orientation is a gradual process, and iteratively solving by using least square method is more accurate. A large number of experiments show that the revision coefficients of all parameters are less than the radian with a threshold value of $0.1 \times 10^{-4}$, the expected accuracy is attained. Then the calculation ends.

\subsection{Steps of Algorithm}

(1) Assign initial values to unit quaternions $a, b, c, d$ and $B_{x}, B_{y}, B_{z}, f$, among which $B_{x}$ is used to determine the ratio of the model, $f$ is the focal length of interior orientation.

(2) Calculate the rotation matrix $\mathrm{R}$ formed by unit quaternion by using formula (6).

(3) Substitute the matched image pairs into formula (2) to calculate $X_{1}, Y_{1}, Z_{1}, X_{2}, Y_{2}, Z_{2}$; calculate the error coefficients of matched image pairs by using formula (21) and normalize the error modes one by one.

(4) Solve the normal equation and substitute the solutions $\varepsilon_{1}, \varepsilon_{2}, \varepsilon_{3}$ into formula (11); Combine with formula (12) to solve the correction values $\Delta a, \Delta b, \Delta c, \Delta d$ of quaternion and update the elements $B_{Y}, B_{Z}, a, b, c, d, f$ to be solved.

(5) Judge the correction values $\Delta a, \Delta b, \Delta c, \Delta d, d B_{Y}, d B_{z}, d f$, if they are all less than radian with a threshold value of $0.1 \times 10^{-4}$, then the algorithm ends; otherwise, go to step (2).

\section{Experiments and Analysis}

In order to compare the variable focal length calibration performance of traditional fixed and variable focal length relative orientation calibration algorithm with $\mathrm{p}$-h algorithm proposed in this paper, two experiments are conducted. One experiment simulates a cube model (as shown in Figure 1) to compare the performance of the above 
mentioned algorithms, and another one applies $\mathrm{p}$-h algorithm to three-dimensional reconstruction of real scenario.

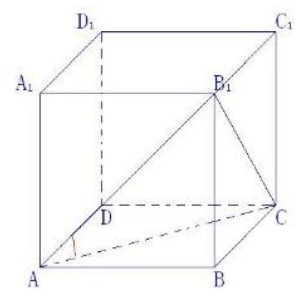

Figure 1. Cube Model

\subsection{Simulation Data}

In the experiment, eight vertexes of the cube model in Figure 1 are simulated, the three-dimensional simulation data of which is shown in Table 1. Simulate the imaging process of the camera by making use of the three -dimensional spatial data to obtain two simulated images, the two-dimensional discretization coordinates of which are shown in Table 2.

Table 1. Three -dimensional Spatial Data of Cube Vertexes

\begin{tabular}{c|c|c|c|c|c|c|c}
\hline No & $\mathrm{X}$ & $\mathrm{Y}$ & $\mathrm{Z}$ & No & $\mathrm{X}$ & $\mathrm{Y}$ & $\mathrm{Z}$ \\
\hline $\mathrm{A}$ & 0 & 0 & 0 & $\mathrm{~A} 1$ & 0 & 1000 & 0 \\
\hline B & 1000 & 0 & 0 & $\mathrm{~B} 1$ & 1000 & 1000 & 0 \\
\hline $\mathrm{C}$ & 1000 & 0 & 1000 & $\mathrm{C} 1$ & 1000 & 1000 & 1000 \\
\hline $\mathrm{D}$ & 0 & 0 & 1000 & $\mathrm{D} 1$ & 0 & 1000 & 1000 \\
\hline
\end{tabular}

Table 2. Simulation Image Data

\begin{tabular}{c|c|c|c|c}
\hline \multirow{2}{*}{$\begin{array}{c}\text { 2-D image } \\
\text { pair }\end{array}$} & \multicolumn{2}{|c|}{ Left image } & \multicolumn{2}{c}{ Right image } \\
\cline { 2 - 5 } A & $\mathrm{x} / \mathrm{pxel}$ & $\mathrm{y} / \mathrm{pxel}$ & $\mathrm{x} / \mathrm{pxel}$ & $\mathrm{y} / \mathrm{pxel}$ \\
\hline $\mathrm{A} 1$ & 186 & 169 & 160 & 150 \\
\hline $\mathrm{B}$ & 186 & 203 & 167 & 187 \\
\hline B1 & 220 & 169 & 196 & 146 \\
\hline $\mathrm{C}$ & 219 & 203 & 201 & 182 \\
\hline $\mathrm{C} 1$ & 229 & 158 & 201 & 128 \\
\hline $\mathrm{D}$ & 179 & 158 & 207 & 183 \\
\hline D1 & 180 & 209 & 144 & 132 \\
\hline & & & & 190 \\
\hline
\end{tabular}

\subsection{Results and Analysis}

In simulative experiment, misconvergence exists both in traditional fixed focal length calibration algorithm and variable focal length calibration algorithm, and convergence only can be obtained by repeatedly adjusting the initial value. As for the algorithm proposed in this paper, the initial value is set as $a=b=c=0, d=1, B x=200, \quad B y=0$, $\mathrm{Bz}=0$, and the focal length $f$ is a random number between $80 \%^{f} \sim 120 \%^{f}$ of the simulative camera focal length.

To compare and quantitatively analyze the reconstruction results of $\angle B_{1} A C$ by using the above three algorithms, $\varphi=\omega=0$ is set in the experiments. Parameters $\kappa$ in the left 
image is between -60 and 60 , while in the right image $\kappa$ takes the value of zero and $f$ varies from $80 \%^{f}$ to $120 \%^{f}$. Part of the experiment result is shown in Table 3 .

Table 3. Simulation Experiments Results

\begin{tabular}{|c|c|c|c|c|c|c|c|c|c|}
\hline \multirow[t]{2}{*}{ No. } & \multirow[t]{2}{*}{$\begin{array}{c}\kappa \text { left(degr } \\
\text { ee) }\end{array}$} & \multirow[t]{2}{*}{$\begin{array}{c}\kappa \text { right }(\mathrm{d} \\
\text { egree })\end{array}$} & \multirow[t]{2}{*}{$f$} & \multicolumn{2}{|c|}{$\begin{array}{l}\text { Fixed Focal Length } \\
\text { Relative Orientation } \\
\text { Calibration }\end{array}$} & \multicolumn{2}{|c|}{$\begin{array}{c}\text { Variable Focal Length } \\
\text { Relative Orientation } \\
\text { Calibration }\end{array}$} & \multicolumn{2}{|c|}{$\begin{array}{l}\text { Relative Orientation Based on p-h } \\
\text { Algorithm }\end{array}$} \\
\hline & & & & iterations & $\angle B_{1} A C$ & iterations & $\angle B_{1} A C$ & iterations & $\angle B_{1} A C$ \\
\hline 1 & -60 & 0 & 80 & divergence & divergence & divergenc & divergence & 21 & 60.0141 \\
\hline 2 & -45 & 0 & 95 & 19 & 60.0303 & 17 & 60.021 & 8 & 60.0217 \\
\hline 3 & -30 & 0 & 110 & 9 & 60.0305 & 10 & 60.1055 & 9 & 60.0353 \\
\hline 4 & -21 & 0 & 80 & 7 & 60.0311 & 9 & 60.0787 & 5 & 60.1258 \\
\hline 5 & -12 & 0 & 95 & 4 & 60.0311 & 6 & 60.0307 & 3 & 60.0338 \\
\hline 6 & -3 & 0 & 110 & 5 & 60.0303 & 6 & 60.0585 & 3 & 60.0347 \\
\hline 7 & 6 & 0 & 80 & 4 & 60.0321 & 6 & 60.0318 & 3 & 60.0301 \\
\hline 8 & 24 & 0 & 110 & 8 & 60.0305 & 12 & 60.0874 & 5 & 60.0383 \\
\hline 9 & 35 & 0 & 80 & 10 & 60.0322 & 10 & 60.2449 & 5 & 59.9788 \\
\hline 10 & 55 & 0 & 100 & divergence & divergence & divergenc & divergence & 7 & 59.9660 \\
\hline
\end{tabular}

According to the simulation experiment results, we get the following findings.

(1)Parameter initialization: traditional relative orientation algorithm and variable focal length relative orientation algorithm depend strongly on the initial value and require the initial value be as close as possible to the true value; otherwise, it may cause misconvergence in the algorithms. The new algorithm just set the rotation matrix as $a=b=c=0$ and $d=1$ to get accurate results. Therefore, the new algorithm with variable focal length relative orientation algorithm proves to have strong adaptation.

(2)Iterations: there are 4.75 iterations on average in variable focal length relative orientation algorithm based on p-h algorithm, which is less than the other two algorithms. This shows that the new algorithm have advantages of less iterations and faster convergence by using $\mathrm{p}$-h algorithm to obtain optimization solution.

(3)Accuracy analysis: in the respect of rebuilding angle accuracy, three algorithms are all convergent. However, the accuracy of rebuilding $\angle B_{1} A C$ with traditional fixed focal length relative orientation algorithm and variable focal length relative orientation algorithm based on p-h algorithm are about the same, and it is higher than that of variable focal length relative orientation algorithm.

(4)Rotation angle: if the difference of the rotation angles in two images is large, the traditional relative orientation algorithm and variable focal length relative orientation algorithm cannot reach convergence, while the new algorithm is able to give rational solution. For instance, in Table 3, when $\mathrm{k}=-60$, convergence is available after 21 iterations and the rebuilt angle is 60.0141. This result shows that the new algorithm has the advantage of reaching convergence with wide angle.

(5)Multivaluedness: the new algorithm adopt $\mathrm{p}-\mathrm{h}$ algorithm to optimize the matrix derivation and form the rotation matrix by replacing euler angle with unit quaternion. This effectively solves the problems of multivaluedness of trigonometric function and singularity based on trigonometric function rotation matrix.

\subsection{Rebuild the Real Scenario}

To verify the feasibility and validity of the new algorithm in rebuilding the real scenario, three-dimensional reconstruction simulation experiment is conducted as illustrated in Figure 2. 


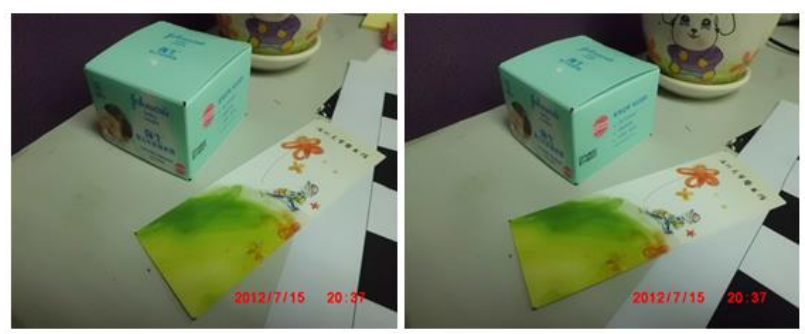

Figure 2. Images to be Rebuilt

In the experiment, the algorithm proposed by Zhang Zhengyou is adopted to calibrate the intrinsic parameters of the camera; the SRUF algorithm [12] is used to extract the feature points and conduct matching calculation; seven pairs of synonymous image pairs are picked and calibrated by the algorithm proposed in this paper.

In the end, the following parameters are obtained: $\mathrm{a}=-0.216568, \mathrm{~b}=-0.282970$, $\mathrm{c}=0.084564, \mathrm{~d}=-0.956597, \mathrm{By}=-69.674440, \quad \mathrm{Bz}=482.207348 f=476.991886$, and the rotation matrix formed by quaternion is as follows.

$$
\left[\begin{array}{ccc}
0.825554 & -0.0392228 & -0.578004 \\
0.284352 & 0.891894 & 0.366478 \\
0.504749 & 0.462195 & 0.746053
\end{array}\right]
$$

By using formula (7), we may obtain three rotation angles $\varphi_{\omega \kappa}:^{\varphi}=0.659154, \omega=-$ $0.375222, \quad \kappa=-0.308631$.

We use forward intersection algorithm [13] to calculate three-dimensional coordinates of the matched synonymous image points, then we can simulate all real scenario with OpenGL technology. The simulation result is illustrated in Figure 3, which shows the rotation results under different angles after rebuilding.
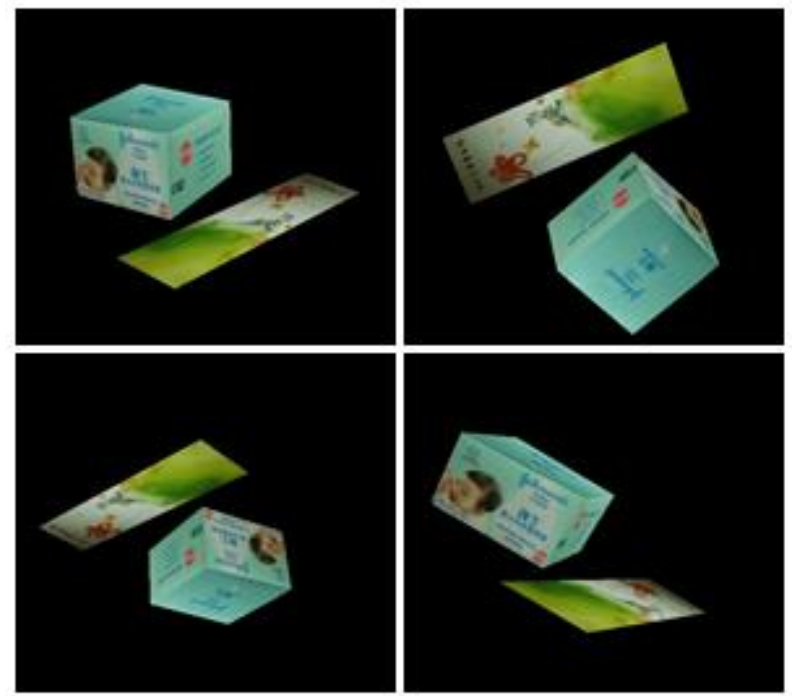

Figure 3. Simulation Result

The simulation result shows that ideal rebuilding effect can be achieved by adopting variable focal length relative orientation method based $\mathrm{p}$-h algorithm. 


\section{Conclusion}

The traditional relative orientation calibration algorithm describes the rotation matrix by using trigonometric function, and problems like more iterations, strong dependence of initial value and misconvergence of wide angles exist in solution procedure. The algorithm proposed in this paper replaces trigonometric function with unit quaternion to form rotation matrix and optimize the derivation process with $\mathrm{p}$-h algorithm. The simulation result shows that the new algorithm has the advantages of less dependence, fast convergence, less iterations and great flexibility of wide angles compared with traditional relative orientation calibration algorithm. Moreover, this algorithm can effectively eliminate multivaluedness and singularity in rotation matrix formed by trigonometric function.

\section{References}

[1] R. Liu, J. Gong and J. Tian, "Calibration Technology of Line-scan Cameras Based on Quadratic Cuves [J]”, Computer Engineering, vol. 39, no. 1, (2013), pp. 283-286.

[2] R. Y. Tsai, "A versatile camera calibration technique for high-accuracy 3D machine vision metrology using off-the shelf tv cameras and lenses [Z]", IEEE Journal of Robotics and Automation, vol. 3, no. 4, (1987), pp. 323-344.

[3] Z. Zhang, "A flexible new technique for camera calibration [J]", IEEE Transaction on Pattern Analysis and Machine Intelligence, vol. 22, no, 11, (2000), pp. 1330- 1334.

[4] Y. Zhang and B. Hu, "Relative Orientation Based on Multiple Conjugate Features [J]", Acta Geodaeticaet Cartographica Sinica, vol. 40, no. 2, (2011), pp. 194-199.

[5] Y. Lu, Y. Chen and B. Zheng, "Research on dependent relative orientation in Multi-baseline Close-range photogrammetry [J]", Journal of Tongji University, vol. 38, no. 3, (2010), pp. 442-447.

[6] H. Cao and R. Wang, "The application of Random Sample Consensus in photogrammetric relative orientation [C]", ICIECS, (2010), pp. 4113-4116.

[7] L. Yang, M. MA and L. Miao, "Relative Orientation Method for the Close-Range Digital imagery of Non-Metric Camera [J]", Journal of Geodesy and Geodynamics, vol. 32, no. 4, (2012), pp. 135-138.

[8] J. Zhao, K. Zhang and X. Wei, "An improved relative orientation Calibration Method [J]", Science Technology and Engineering, vol. 13, no. 32, (2013), pp. 9744-9748.

[9] A. Pope, "An Advantageous, Alternative Parametzation of Rotations for Analytical Photogrammetry", ESSA Technical Report CaGS39, Rockville, Maryland, USA, (1970).

[10] L. Hinsken, "A singularity free algorithm for spatial orientation of bundles [J]", International Archives of Photogrammetry and Remote Sensing, vol. 27, no. B5, (1988), pp. 262-272.

[11] Y. Wang, T. Jiang and G. Jiang, "Space Resection of Single Image Based on the Description of Unit Quaternions [J]", Journal of Geomatics Science and Technology, vol. 24, no. 2, (2007), pp. 133-135.

[12] H. Bay, T. Tuytelaars and L. Van Gool, "SURF: Speeded up robust features [C]", Proceedings of the European Conference on Computer Vision, (2006), pp. 404-407.

[13] W. Wang and F. An, "Accuracy comparison of different space intersection models based on generalized stereopair [J]", Science of Surveying and Mapping, vol. 37, no. 2, (2012), pp. 10-13.

\section{Authors}

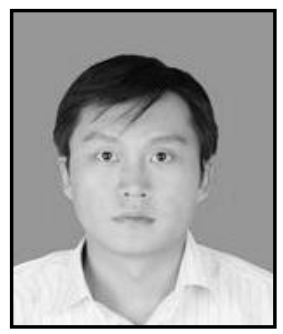

Bin Yang, He received his M.Sc. in Computer Science (2009) from Xidian University. Now he is lecturer of computer science at Institute of Computer Science and Engineering, Henan University of Urban Construction. His current research interests include different aspects of computer application, digital media technology and computer network. 


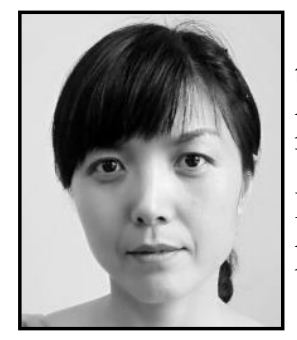

Yongjiao Wang, She received her master's degree in computer technology (2005) from Zhejiang University. Now she is an associate professor in School of Computer Science, University of Urban Construction, Pingdingshan, city, P.R. China. Her main research area focused on the Optical fiber sensor and image processing.

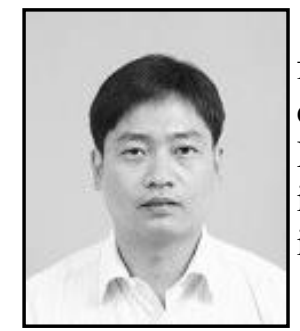

Junmin Zhao, He received his M.Sc. in Computer Science (2008) from Henan University of Finance and Law. Now he is lecturer of computer science at Institute of Computer Science and Engineering, Henan University of Urban Construction. His research interest includes computer application, bioinformatics and management information system. 
International Journal of Control and Automation Vol.8, No.5 (2015) 